\title{
Genetics of aliphatic glucosinolates. III. Side chain structure of aliphatic glucosinolates in Arabidopsis thaliana
}

\author{
R. MITHEN*, J. CLARKE†, C. LISTER $†$ \& C. DEAN $\dagger$ \\ Brassica and Oilseeds Research Department and + Molecular Genetics Department, John Innes Centre, Colney Lane, \\ Norwich NR4 7UJ, U.K.
}

\begin{abstract}
The inheritance of aliphatic glucosinolates in Arabidopsis thaliana was studied. Analysis of $\mathrm{F}_{3}$ families from a cross between the ecotype Limburg-5 and the accession H51 (an inbred line derived from the ecotype Stockholm) suggested that alleles at a single locus regulate the conversion of methylsulphinylalkyl glucosinolates into alkenyl (and hydroxyalkenyl) glucosinolates. Likewise, analysis of recombinant inbred lines derived from a cross between the ecotypes Columbia and Landsberg erecta suggested that alleles at a single locus regulate the conversion of methylsulphinylpropyl glucosinolate into hydroxypropyl glucosinolate. Both loci mapped to a similar position on chromosome 4 and it is suggested that these alleles occur at the same locus. A genetic model is proposed in which the aliphatic glucosinolates of $A$. thaliana are determined by alleles at three loci. The ecological significance of variation in aliphatic glucosinolates is discussed.
\end{abstract}

Keywords: Arabidopsis thaliana, gene mapping, genetic diversity, glucosinolates, pests.

\section{Introduction}

Aliphatic glucosinolates are thioglycosides which occur in the leaves of members of the Brassicaceae and related families. They are important in mediating pest interactions and have been shown to attract specialized cruciferous pests such as flea beetles and to repel generalist herbivores such as slugs and birds. In addition, they determine the feeding quality of rapeseed meal and the flavour of cruciferous vegetables and salad crops. The aliphatic glucosinolate molecule comprises two parts: a common glycone moiety and a variable aglycone side chain derived from methionine (Fenwick et al., 1983). The biological activity of glucosinolates is largely dependent on the structure of the side chain which determines the nature of hydrolysis products which are produced following tissue damage. Intraspecific variation in the types of aliphatic glucosinolates has been shown to occur in Cakile edentula (Rodman, 1980), Brassica oleracea (sensu lato) (Mithen et al., 1987) and Arabidopsis thaliana (Bano, 1993). This variation may be in the length of the aliphatic side chain or in the types of substituted groups which it may possess, both of which influence pest interactions

\footnotetext{
*Correspondence.
}

(Chew, 1988; Mithen 1992; Simmonds et al., 1994). In A. thaliana, methylsulphinylalkyl, alkenyl, hydroxyalkyl and hydroxyalkenyl homologues of propyl and butyl glucosinolates are commonly found (Bano, 1993, fig. 1), with trace levels of longer side chain homologues.

In a previous publication, it was shown that the side chain length of aliphatic glucosinolates in $A$. thaliana is regulated by alleles at a single locus on chromosome 5 (Magrath et al., 1994). In the present study, we consider two major types of side chain modifications. Firstly, a desaturation reaction which converts methylsulphinylalkyl glucosinolates into alkenyl glucosinolates (Fig. 2), and secondly, the production of hydroxypropyl glucosinolate. A third type of side chain modification, the addition of hydroxyl group to butenyl glucosinolate will be considered separately. A model of aliphatic glucosinolate biosynthesis is developed which is used to explain the diversity of aliphatic glucosinolates found in $A$. thaliana ecotypes.

\section{Materials and methods}

To investigate the conversion of methylsulphinylalkyl glucosinolates into alkenyl glucosinolates, a cross was made between the ecotype Limburg-5, which has pre- 


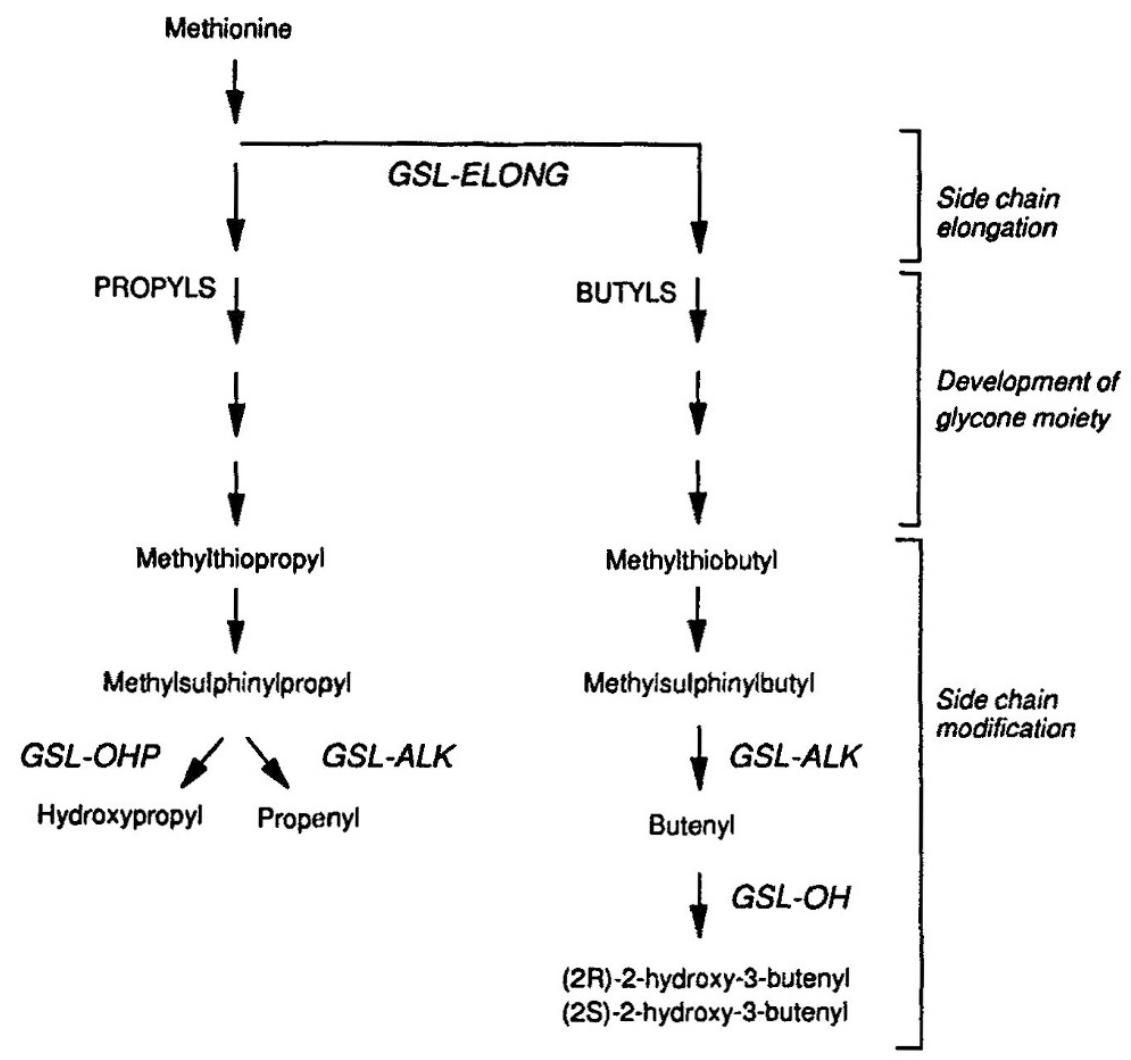

Fig. 1 Genetic model of side chain elongation and modification of aliphatic glucosinolates in Arabidopsis thaliana. dominantly methylsulphinylbutyl and methylsulphinylpropyl glucosinolates and the inbred line H51 (a selection from the ecotype Stockholm) which has predominantly butenyl, hydroxybutenyl and propenyl glucosinolates (Fig. 2). The $F_{1}$ was selfed and $F_{2}$ and $F_{3}$ families generated, as described by Clarke \& Dean (1994). All plants were grown under standard conditions as described previously (Magrath et al., 1994). Glucosinolates were extracted from freeze-dried leaf tissue, converted to desulphoglucosinolates and analysed by HPLC as previously described (Magrath $e t$ al., 1993). To investigate the production of hydroxypropyl glucosinolate, the segregation of glucosinolate profiles in recombinant inbred (RI) lines from a cross between Landsberg erecta, which has predominantly hydroxypropyl glucosinolate, and Columbia, which lacks this glucosinolate (Fig. 3) was examined, as described previously (Magrath et al., 1994). RFLP mapping in the cross between Limburg-5 and H51 was as described by Clarke \& Dean (1994) and in the cross between Landsberg erecta and Columbia as described by Lister \& Dean (1993). The glucosinolate profiles of $\mathrm{F}_{1}$ hybrids between Columbia and $\mathrm{H} 51$, and between the ecotypes Kil-0 and Columbia were also examined.

\section{Results}

\section{Cross 1: desaturation}

The bulk tissue samples from the $F_{3}$ families had two distinct profiles which corresponded to the parental phenotypes (Fig. 2). Twenty-five families and the $F_{1}$ hybrid possessed predominantly butenyl, hydroxybutenyl and propenyl glucosinolates (profile (b), Fig. 2) whereas 17 families possessed methylsulphinylbutyl and methylsulphinylpropyl glucosinolates (profile (a), Fig. 2), corresponding to the two parental phenotypes. The ratio is not significantly different from 3:1 $\langle 0.05>P>0.01)$ suggesting the action of a dominant allele at a single locus $(G S L-A L K\rangle$ which converts methylsulphinylalkyl glucosinolates into alkenyl glucosinolates. RFLP mapping places this locus between RFLP markers g3843 and w167 on chromosome 4 (Fig. 4).

\section{Cross 2: hydroxylation of propyl glucosinolates}

Four different glucosinolate profiles were found within the RI lines. Two of the profiles corresponded to the parental phenotypes (Fig. 3) and two were novel phenotypes, one of which contained methylsulphinylbutyl and hydoxypropyl glucosinolates (Fig. 5a) and 

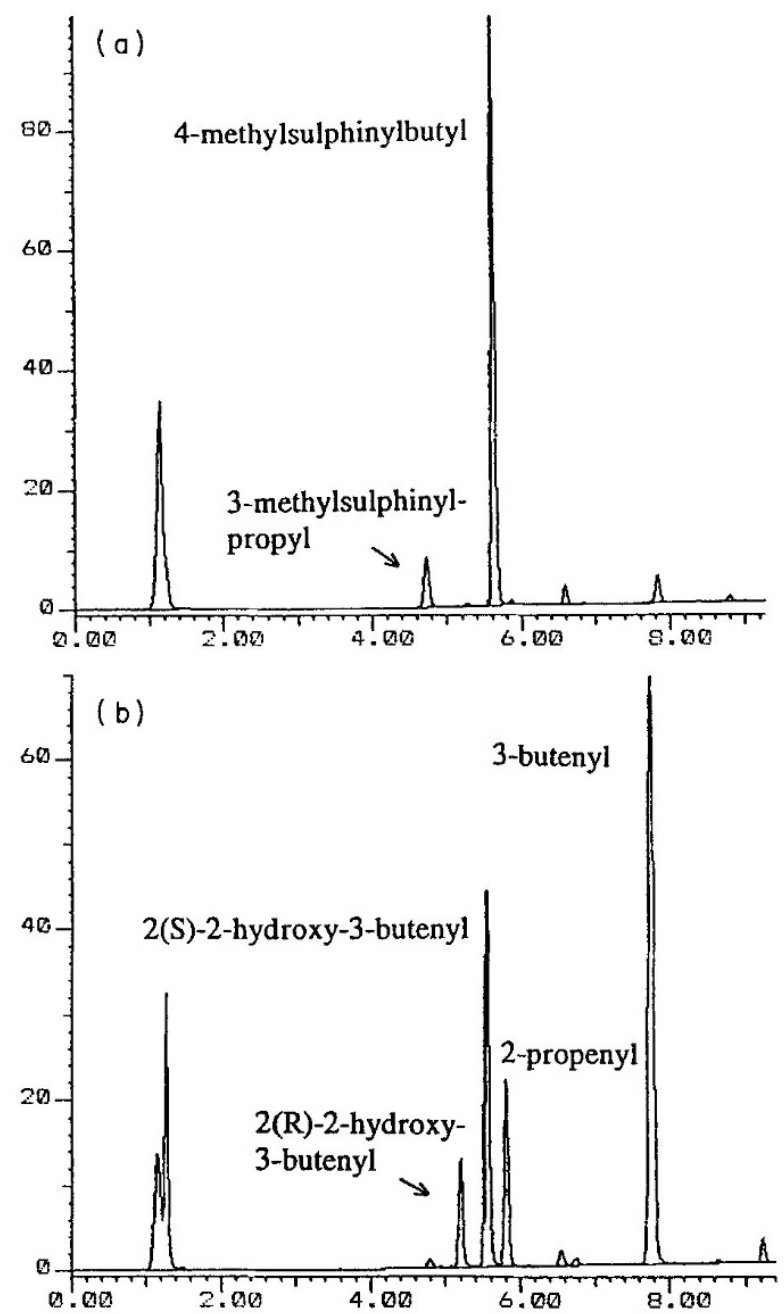

Fig. 2 Conversion of methylsulphinylalkyl glucosinolates to alkenyl glucosinolates in Arabidopsis thaliana. Limburg-5 has profile (a) and $\mathrm{H} 51$ profile (b). All recombinants corresponded to the two parental phenotypes.

the other only methylsulphinylpropyl glucosinolate (Fig. 5b). The presence of methylsulphinylbutyl glucosinolate results from the inheritance of the Columbia allele at the GSL-ELONG locus described by Magrath et al. (1994). Fifty-three of the RI lines had significant amounts of hydroxypropyl glucosinolate whereas the remaining 47 lines had no detectable amounts of this glucosinolate. The ratio is not significantly different from a 1:1 $(P>0.05)$ suggesting the action of two alleles at a single locus (GSL-OHP). The proportion of hydroxypropyl glucosinolate depended on the presence of alleles at the GSL-ELONG locus (Magrath et al., 1994); RI lines with the Landsberg erecta allele at this locus had 100 per cent hydroxypropyl glucosinolate whereas if the RI lines possessed the Columbia allele at this locus the proportion of hydroxypropyl glucosinolate fell to less than 10 per cent of the total aliphatic glucosinolate content (Fig. 5). RFLP mapping places this locus between RFLP markers w390 and m448 on chromosome 4 , in a similar position to the $G S L-A L K$ locus mapped in the first cross.

Hydroxypropyl glucosinolate may be derived from propenyl glucosinolate, in an analogous manner to which hydroxybutenyl glucosinolate is derived from butenyl glucosinolate. Hence if Columbia carried a 'silent' allele for hydroxylation, the locus mapped in cross 2 may regulate the desaturation of methylsulphinylpropyl glucosinolate, i.e. the same locus that was mapped in cross 1 . To test this hypothesis, the glucosinolate profiles of two $F_{1}$ hybrids were examined, first that between Columbia and $\mathrm{H} 51$, and secondly between Columbia and the ecotype Kil-0, which only produced propenyl glucosinolate (Fig. 3). If Columbia carried a silent GSL-OHP allele, hydroxypropyl would be expected to occur in both of these $F_{1}$ hybrids. However, in the $F_{1}$ hybrids there was no evidence for this glucosinolate (Fig. 3). Therefore it appears that the gene mapped in cross 2 does regulate the hydroxylation of propyl glucosinolate. In $F_{1}$ hybrids between Columbia and Kil-0, hydroxybutenyl glucosinolate is found suggesting that one or both of the parents carry functional alleles for the hydroxylation of butenyl glucosinolate (Fig. 3). Hydroxybutenyl glucosinolate does not occur in either parent as both parents lack the precursor butenyl glucosinolate; Kil-0 as it lacks a functional allele at the GSL-ELONG locus and Columbia as it lacks a functional allele at the GSL$A L K$ locus (Fig. 1).

The functional $G S L-A L K$ and $G S L-O H P$ alleles may occur at two linked loci or may be different alleles at the same locus. A third allele at this locus (such as that which occurs in Columbia) would not result in any modification of methylsulphinylalkyl glucosinolates.

\section{Discussion}

The results indicate that two important side chain modifications of glucosinolates in $A$. thaliana are regulated by alleles on chromosome 4; firstly, the conversion of methylsulphinylalkyl to alkenyl glucosinolates and, secondly, the conversion of methylsulphinylpropyl glucosinolate to hydroxypropyl glucosinolate. It is likely that these alleles are at the same locus or are at two closely linked loci. A third allele, such as that found in Columbia, does not result in any modification of methylsulphinylalkyl glucosinolates. If the $G S L$ $A L K$ gene were cloned it is possible that it could be used in antisense experiments in Brassica, either directly or by isolating the Brassica homologue. This should prevent the desaturation of methyl- 

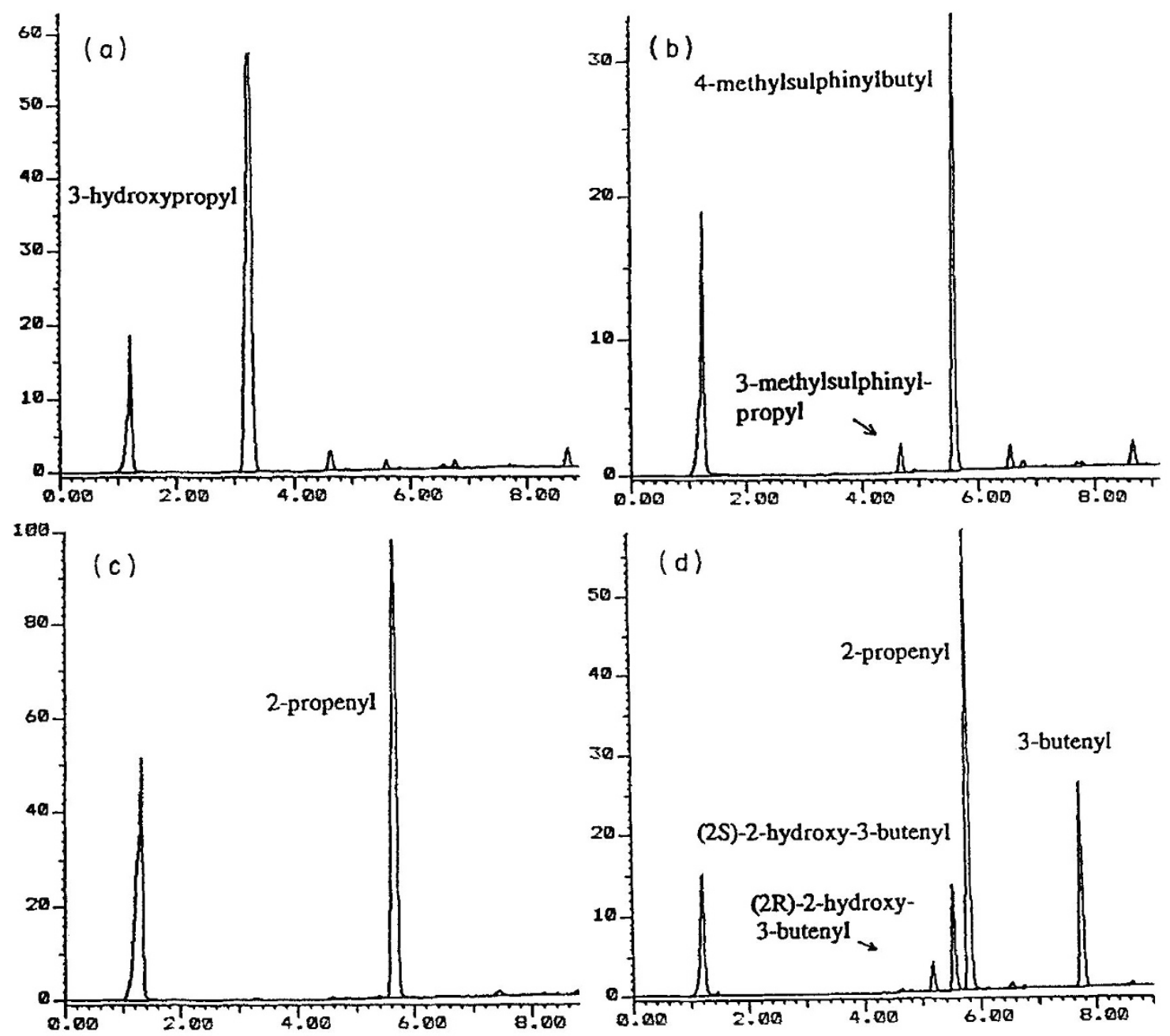

Fig. 3 Aliphatic glucosinolates of Arabidopsis thaliana Landsberg erecta (a), Columbia (b), Kil-0 (c) and the $\mathrm{F}_{1}$ hybrid between Columbia and Kil- 0 (d). The absence of hydroxypropyl glucosinolate in the $F_{1}$ hybrid suggests that Columbia does not carry a 'silent' allele at a putative GSL-OHP locus. Details of aliphatic glucosinolates in RI lines from a cross between Landsberg erecta and Columbia are given by Magrath et al. (1994).

sulphinylalkyl glucosinolates which would limit the production of hydroxyalkenyl glucosinolates, which are the major toxic components of rapeseed meal. This would provide an alternative approach to increasing the quality of rapeseed meal to that described by Parkin et al. (1994).

The variation in profiles observed in these ecotypes of $A$. thaliana can be explained by the simple genetic model shown in Table 1, in which the glucosinolates which are found in leaf tissue are determined by alleles at three loci: GSL-ELONG which regulates side chain elongation and is on chromosome 5 (Magrath et al., 1994), GSL-ALK/OHP which regulates the conversion of methylsulphinylalkyl to alkenyl glucosinolates and the production of hydroxypropyl glucosinolate and is on chromosome 4 (this study), and a third unmapped locus (unpublished data), designated GSL$\mathrm{OH}-\mathrm{Ar}$, which regulates the hydroxylation of butenyl glucosinolate, analogous to the GSL-OH-A and GSL$\mathrm{OH}-\mathrm{C}$ loci found in Brassica napus (Parkin et al., 1994). Combination of functional and null alleles at these three loci (Table 1) explains the variation in aliphatic glucosinolates found within the 63 ecotypes of $A$. thaliana described by Bano (1993). The absence of ecotypes with both propenyl and hydroxypropyl glucosinolates supports the hypothesis that the GSL$A L K$ and GSL-OHP alleles occur at the same locus. Both glucosinolates would only occur if the individual examined at this locus was heterozygous for these two alleles, which is unlikely considering the high degree of inbreeding which occurs in $A$. thaliana. A similar model explains the variation of aliphatic glucosinolates 


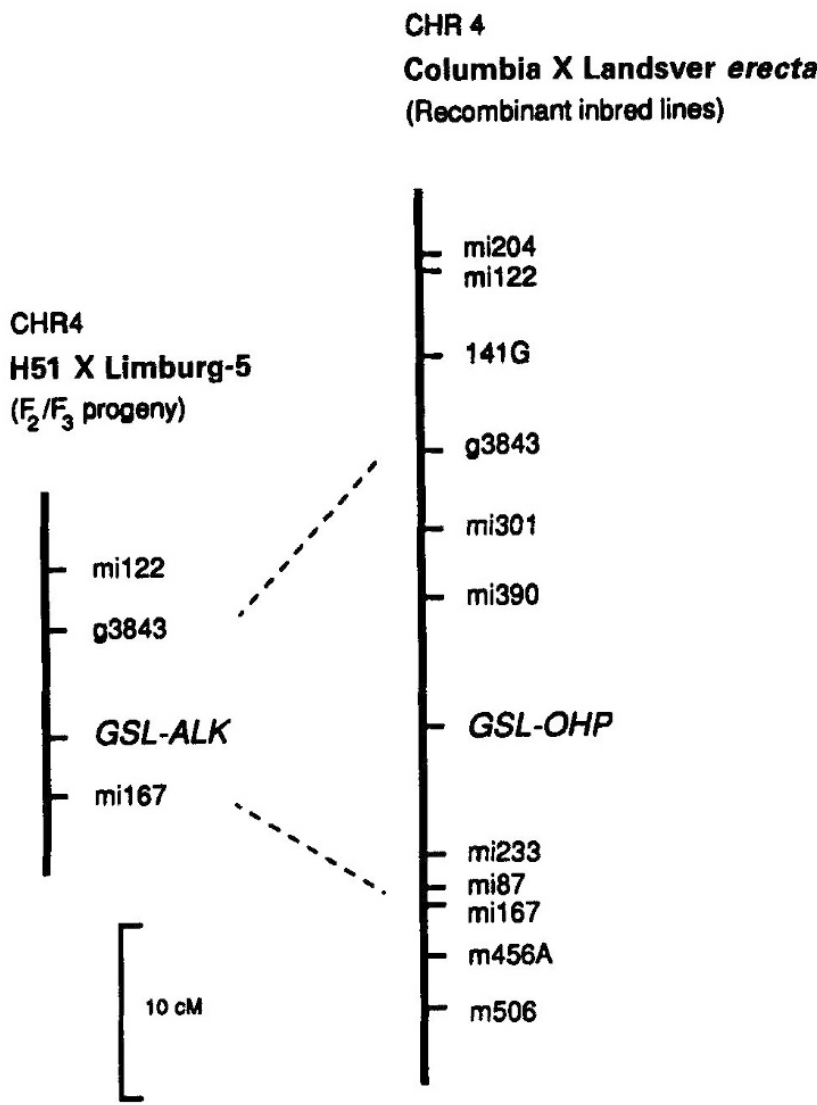

Fig. 4 RFLP maps of the positions of the $G S L-A L K$ and GSL-OHP loci of Arabidopsis thaliana, mapped in two separate crosses. Both loci map to the same interval.
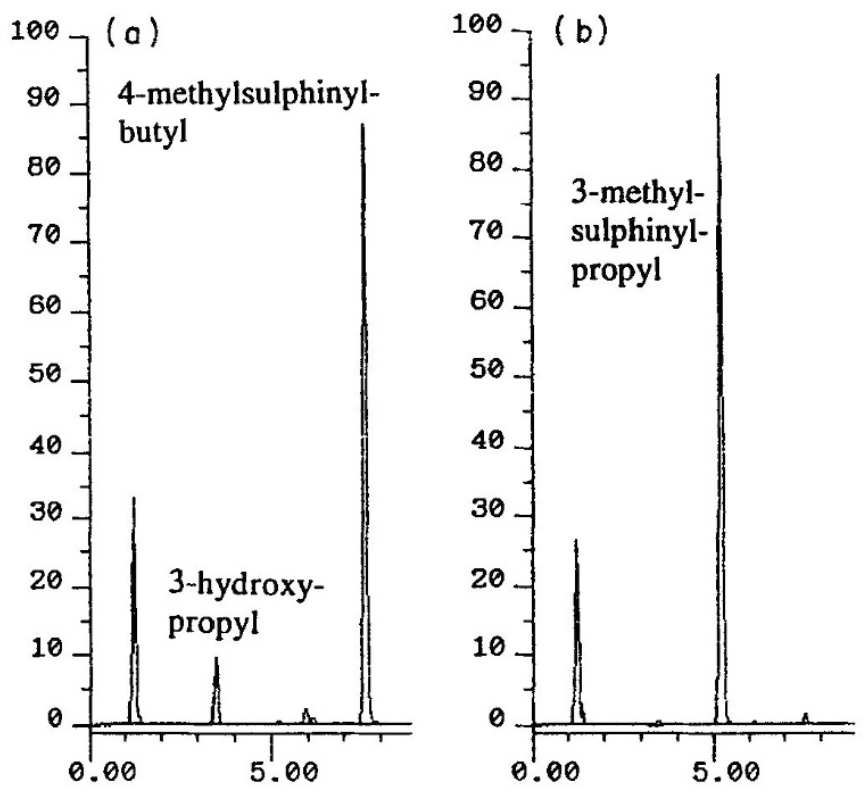

Fig. 5 Novel aliphatic glucosinolate profiles in $\mathrm{RI}$ lines of Arabidopsis thaliana from a cross between Landsberg erecta and Columbia. Genotypes with profiles (a) combine the Columbia allele at the GSL-ELONG locus with the Landsberg erecta allele at the GSL-OHP locus whereas genotypes with profile (b) combine the Landsberg erecta allele at the GSL-ELONG locus with the Columbia allele at the GSL-OHP locus. Further information is given in Table 1.

Table 1 Genetic model for the phenotypic expression of aliphatic glucosinolates Arabidopsis thaliana. The alk and ohp alleles occur at the same locus (or at two loci which are closely linked) on chromosome 4 . The model explains the variation in aliphatic glucosinolates reported to occur in A. thaliana ecotypes (Bano, 1993). Only homozygote genotypes are shown

\begin{tabular}{|c|c|c|c|}
\hline \multicolumn{2}{|c|}{ Locus } & \multirow{2}{*}{ Phenotype } & \multirow{2}{*}{ Example } \\
\hline GSL-ELONG & $G S L-A L K / O H P$ & & \\
\hline & & & \\
\hline elong/elong & alk/alk & $\begin{array}{l}\text { Butenyl ( } \pm \text { hydroxylation), } \\
\text { propenyl }\end{array}$ & H51 (Fig. 2b) \\
\hline elong/elong & ohp/ohp & $\begin{array}{l}\text { Methylsulphinylbutyl + } \\
\text { hydroxypropyl }\end{array}$ & RI 194 (Fig. 5a) \\
\hline elong/elong & null/null & $\begin{array}{l}\text { Methylsulphinylbutyl + } \\
\text { methylsulphinylpropyl }\end{array}$ & $\begin{array}{l}\text { Limburg-5 (Fig. 2a) } \\
\text { Columbia (Fig. 3b) }\end{array}$ \\
\hline null/null & alk/alk & Propenyl & Kil-0 (Fig. 3c) \\
\hline null/null & ohp/ohp & Hydroxypropyl & Landsberg erecta (Fig. 3a) \\
\hline null/null & null/null & Methylsulphinylpropyl & RI 191 (Fig. 5b) \\
\hline
\end{tabular}


in Brassica (Magrath et al., 1994; Parkin et al., 1994; A. Giamoustaris and R. Mithen, unpublished data) and is likely to be applicable to the genetic regulation of aliphatic glucosinolates in other crucifers. This enables variation of aliphatic glucosinolates within and between wild crucifer populations to be interpreted in terms of the frequency of a small number of alleles.

Aliphatic glucosinolates mediate pest and pathogen interactions with crucifers. They decrease the palatability of leaf tissue to nonspecific herbivores such as slugs and birds, but increase the susceptibility of tissue to specialized insect pests such as flea beetles. It has been shown that both side chain length and modifications can affect the nature of these interactions. The differences in aliphatic glucosinolate content may be responsible for the variation in susceptibility of $A$. thaliana ecotypes to pests which is frequently observed when different ecotypes are grown under controlled conditions (personal observations). Likewise, natural populations of $A$. thaliana, Brassica and other crucifers may be exposed to different pest pressures in different environments which may lead to contrasting selection pressures on individuals with different aliphatic glucosinolate content. This may explain the intraspecific variation observed in aliphatic glucosinolate content both within and between populations. Fluctuating pest pressures, both in terms of the types (specialist vs. generalist) and frequency of pests, may result in contrasting selection pressures in different generations which would result in the maintenance of genetic diversity within populations.

\section{Acknowledgements}

The authors thank Dr J. Turner and Dr F. Bano, School of Biological Sciences, University of East Anglia, for discussions concerning glucosinolates in A. thaliana, Dr Robert Whittier for unpublished RFLP markers (mi...) and segregation data on the RI lines, and Ruth Magrath for technical assistance.

\section{References}

BANO, F. 1993. Glucosinolates in Arabidopsis. Ph.D. Thesis, University of East Anglia, Norwich.

CHEW, F. S. 1988. Biological effects of glucosinolates. In: Cutler, H.G. (ed.) Biologically Active Natural Products: Potential Use in Agriculture, pp. 155-181. American Chemical Society Symposium, Washington, DC.

ClARKE, J. H. AND DEAN, C. 1994. Mapping FRI, a locus controlling flowering time and vernalization response in Arabidopsis thaliana. Mol. Gen. Genet., 242, 81-89.

FENWICK, G. R., HEANEY, R. K. AND MULLIN, W. J. 1983. Glucosinolates and their breakdown products in food and food plants. Crit. Rev. Food Sci. Nutr., 18, 123-301.

LISTER, C. AND DEAN, C. 1993. Recombinant inbred lines for mapping RFLP and phenotypic markers in Arabidopsis thaliana. Plant J., 4, 745-750.

MAGRATH, R., HERRON, C., GIAMOUSTARIS, A. AND MITHEN R. 1993. The inheritance of aliphatic glucosinolates in Brassica napus. Pl. Breed., 111, 55-72.

MAGRATH, R., BANO, F., MORGNER M., PARKIN, I., SHARPE, A., LISTER, C., DEAN, C., TURNER, J., LYDIATE, D. AND MITHEN, R. 1994. Genetics of aliphatic glucosinolates. I. Side chain elongation in Brassica napus and Arabidopsis thaliana. Heredity, 72, 290-299.

MITHEN, R. 1992. Leaf glucosinolate profiles and their relationship to pest and disease resistance in oilseed rape. Euphytica, 63, 71-83.

MITHEN, R. F., LEWIS, B. G. AND FENWICK, G. R. 1987. Glucosinolates in wild and cultivated Brassica species. Phytochemistry, 26, 1969-1973.

PARKIN, I., MAGRATH, R., KEITH, D., SHARPE, A., MITHEN, R. AND LYDIATE, D. 1994. Genetics of aliphatic glucosinolates. II. Hydroxylation of alkenyl glucosinolates in Brassica napus. Heredity, 72, 594-598.

RODMAN, J. E. 1980. Population variation and hybridization in sea rockets (Cakile edentula, Cruciferae) on the Outer Banks of North Carolina: seed glucosinolates. Am. J. Bot., 67, 1145-1159.

SIMMONDS, M. S. J., BLANEY, W. M., MITHEN, R., BIRCH, A. N. E. AND LEWIS, J. 1994. Behavioural and chemosensory responses of the turnip root fly (Delia floralis) to glucosinolates. Entomologia exp. appl., 71, 45-57. 